\title{
LAP, a novel member of the C/EBP gene family, encodes a liver-enriched transcriptional activator protein
}

\author{
Patrick Descombes, ${ }^{1}$ Mario Chojkier, ${ }^{1,2}$ Serge Lichtsteiner, Eileen Falvey, and Ueli Schibler \\ Department of Molecular Biology, University of Geneva, 1211 Geneva 4, Switzerland; ${ }^{2}$ Department of Medicine, University \\ of California, San Diego, California 92130 USA
}

\begin{abstract}
A gene, encoding a liver-enriched transcriptional activator protein (LAP) has been isolated. LAP is a 32-kD protein that stimulates the transcription of chimeric genes containing albumin D-promoter elements both in vivo and in vitro. LAP shares extensive sequence homology $(71 \%)$ in its DNA-binding and leucine zipper domains with C/EBP. As a consequence, these two proteins show an indistinguishable DNA-binding specificity and readily heterodimerize. In addition, both genes, lap and cebp, are devoid of intervening sequences. Although correctly initiated transcripts from the LAP gene accumulate in the six examined tissues-liver, lung, spleen, kidney, brain, and testis-LAP protein is highly enriched in liver nuclei. Thus, the preferential accumulation of LAP protein in liver appears to be regulated post-transcriptionally.
\end{abstract}

[Key Words: LAP; DNA-binding protein; C/EBP; transcriptional activation]

Received May 24, 1990; revised version accepted June 21, 1990.

During the development of higher eukaryotes, certain genes become activated in a cell-type-specific manner. At least in part, this tissue-specific gene transcription is controlled by diffusible positive transcription factors that are, themselves, enriched in particular cell types. By binding specifically to their DNA recognition sites within promoter and/or enhancer regions, such proteins can augment the frequency of transcription initiation of their target genes, presumably by facilitating the contacts of one or several components of the transcription apparatus (RNA polymerase II, TFIID) with the promoter (for review, see Ptashne 1988).

The recent development of cell-free RNA polymerase II transcription systems for highly differentiated tissues has rendered tissue-specific gene transcription amenable to biochemical analysis (Gorski et al. 1986). For example, the promoter of the mouse albumin gene, containing $\sim 170$ nucleotides of $5^{\prime}$-flanking sequences directs the in vitro synthesis of transcripts at least 50 -fold more efficiently in liver than in spleen nuclear extracts (Gorski et al. 1986; Maire et al. 1989). Yet, the two extracts use the adenovirus late promoter with a similar efficiency. The same promoter sequences also confer cell-type-specific expression to a chloramphenicol acetyltransferase (CAT) reporter gene in transient transfection studies (Heard et al. 1987). The albumin promoter is composed of a TATA motif and six upstream binding sites (A-F) for nuclear proteins (Lichtsteiner et al. 1987). Two of these elements, B and D, are particularly impor-

${ }^{1}$ Both of these authors contributed equally to this work. tant for the efficient liver-specific in vitro transcription from the albumin promoter. Substitution of either one of these cis-acting elements with unrelated DNA reduces dramatically the transcriptional activity of the albumin promoter in liver nuclear extracts. Moreover, synthetic promoters consisting of a TATA motif and multimeric $\mathrm{B}$ or $\mathrm{D}$ elements are much more active in liver than in spleen nuclear extracts (Maire et al. 1989). Element B is a high-affinity site for the well-characterized transcription factor HNF1, a protein that binds to promoter elements of many liver-specific genes (Cereghini et al. 1988; Courtois et al. 1988; Frain et al. 1989; Lichtsteiner and Schibler 1989). Site D can be recognized by liver-enriched factors that are heat resistant and relatively basic (Lichtsteiner et al. 1987). On induction of liver regeneration with carbon tetrachloride, most, if not all, of the D-site-binding activities detectable by gel retardation assays are strongly down-regulated (Mueller et al. 1990). Likewise, these factors do not accumulate to appreciable levels in poorly differentiated hepatoma cells (P. Maire and U. Schibler, unpubl.). The down-regulation of D-binding activities in rapidly dividing cells, such as hepatomas and regenerating hepatocytes, suggests that all or some of these factors may modulate mitogenesis of hepatocytes.

Recently, full-length cDNAs for two members of the D-site-binding proteins, CCAAT/enhancer-binding protein (C/EBP; Landschulz et al. 1988) and D-binding protein (DBP) (Mueller et al. 1990), have been cloned. Both of these proteins activate transcription from the albumin promoter in cotransfection experiments (Fried- 
man et al. 1989; Mueller et al. 1990/. However, neither C/EBP, which accumulates to maximal levels around birth (Birkenmeier et al. 1989), nor DBP, which accumulates only in adult rats (Mueller et al. 1990), can fully account for the developmental accumulation profile and the complex gel mobility-shift pattern of the major Dbinding activities (Mueller et al. 1990). Here, we report on the isolation of the gene encoding a novel D-binding protein, the liver-enriched transcriptional activator protein (LAP). This transcriptional activator protein shares striking sequence homology with C/EBP in its carboxyterminal region and appears to be a major D-binding activity in rat liver nuclei.

\section{Results}

\section{Isolation and characterization of the LAP gene}

For the reasons mentioned above, we anticipated the presence of at least one transcription factor in addition to DBP or C/EBP binding to the albumin promoter element D. To obtain cDNA clones for these additional Dbinding proteins, a $\lambda$ gt 11 expression library was screened with an end-labeled double-stranded oligonucleotide according to the procedures developed by Singh et al. (1988) and Vinson et al. (1988). As a probe in the Southwestern screening, we used an end-labeled D-site mutant oligonucleotide (mutant 10, see Materials and methods), whose affinity for liver D-binding proteins is significantly higher than that observed with the wildtype D element. Binding assays of filters from ten $15-\mathrm{cm}$ culture dishes containing a total of $\sim 2.5 \times 10^{5}$ recombinant phage plaques yielded four double-positives. Three of these encode $\beta$-galactosidase fusion proteins showing the same DNA-binding specificity as the one observed for rat liver D-site-binding proteins (data not shown).

To compare the three recombinant phages specifying the D-site-binding domains, their DNA inserts measuring $\sim 800,750$, and 600 bp were sequenced. All three share common sequences and therefore appear to be overlapping derivatives of the same mRNA species. A sequence data bank search revealed that the sequence of our cDNA clone has not been reported previously, and we named the novel transcription factor LAP.

By Northern blot analysis of polyadenylated liver RNA, the size of LAP mRNA was estimated to be $1.5 \mathrm{~kb}$ (data not shown). Hence, our longest cDNA insert obtained in the initial screening represents only about half of the mRNA sequence. A cDNA insert of 1438 bp [including $18 \mathrm{~A}$ residues of the poly(A) tail] was obtained by screening an oligo(dT)-primed $\lambda$ gt11 rat liver cDNA library (Mueller et al. 1990) by hybridization. Screening of a genomic rat recombinant DNA library (a generous gift of P. Maire and A. Kahn, INSERM, Paris) with radiolabeled cDNA yielded two overlapping EMBL-3 recombinant phages with DNA inserts spanning a total of $17 \mathrm{~kb}$. A 2.3-kb BamHI-EcoRI restriction fragment hybridizing in a Southern blot experiment with both $5^{\prime}$-proximal and $3^{\prime}$-proximal mRNA sequences was subcloned into the plasmid vector $\mathrm{pBS}+$ and sequenced. The compar- ison of its sequence with that of the cDNA clone revealed that the LAP gene is devoid of introns. The nucleotide sequence shown in Figure 1A includes the LAP gene, and $\sim 80$ nucleotides and 240 nucleotides of $5^{\prime}$ - and 3 '-flanking sequences, respectively. The LAP gene encodes mRNAs with somewhat heterogeneous $5^{\prime}$ termini (see below), containing leader sequences of $\sim 50-90 \mathrm{nu}-$ cleotides, an open reading frame (ORF) of 891 nucleotides that potentially could specify a protein of 297 amino acids $(31.5 \mathrm{kD})$, and a trailer sequence of $\sim 480$ nucleotides.

S1 nuclease mapping and cDNA extension experiments with nuclear and cytoplasmic poly $(A)^{+}$liver RNAs were performed to localize the transcriptional start site(s) within the genomic LAP sequence. Multiple species of S1 nuclease-resistant DNA fragments and cDNA extension products were observed, indicating that transcription initiates at multiple sites within a region of $\sim 30$ nucleotides (data not shown). The region containing these putative transcriptional start sites is indicated in Figure 1A.

\section{$L A P$ and $C / E B P$ bind to the same albumin promoter elements}

A protein data bank search (SWISS-PROT 11) revealed extensive sequence similarity $(71 \%)$ between the carboxy-terminal amino acid sequences of LAP and C/EBP (Fig. 1B). For C/EBP, this region has been shown to contain a basic peptide segment and a leucine zipper structure required for DNA binding and dimerization, respectively (Landschulz et al. 1989). The striking sequence conservation within the carboxy-terminal LAP and C/EBP sequences prompted us to examine three obvious possibilities: (1) Does the common DNA binding specificity of these two proteins apply to sites other than $D$ ? (2) Can LAP dimerize in a way similar to C/EBP? (3) If so, can the two proteins form heterodimers? To prepare large amounts of purified recombinant LAP protein, a phage T7 polymerase overexpression system (Studier et al. 1990) was used (Fig. 2A). Figure 2B shows a comparative DNase I protection analysis of purified recombinant LAP and C/EBP on the albumin promoter. Both proteins occupy the same sites of the albumin promoter with a similar preference; they have a high affinity for site $D$, a somewhat lower affinity for sites $A$ and $F$, and fill the same additional sites at higher protein to DNA ratios. From these results we conclude that both proteins have a similar, relatively relaxed binding specificity, suggesting that the few amino acid differences within their basic regions do not significantly affect their DNA recognition. Incubation of purified recombinant LAP in the presence of low concentrations of glutaraldehyde (Landschulz et al. 1989) leads to cross-linked molecules with a size expected for a LAP dimer (Fig. 2C, left). As shown in Figure 2C (right) coincubation of LAP with a peptide containing the carboxy-terminal region of C/EBP in the presence of glutaraldehyde results in the formation of cross-linked molecules with a size expected for LAP-C/EBP dimers. These heterodimers appear to be 


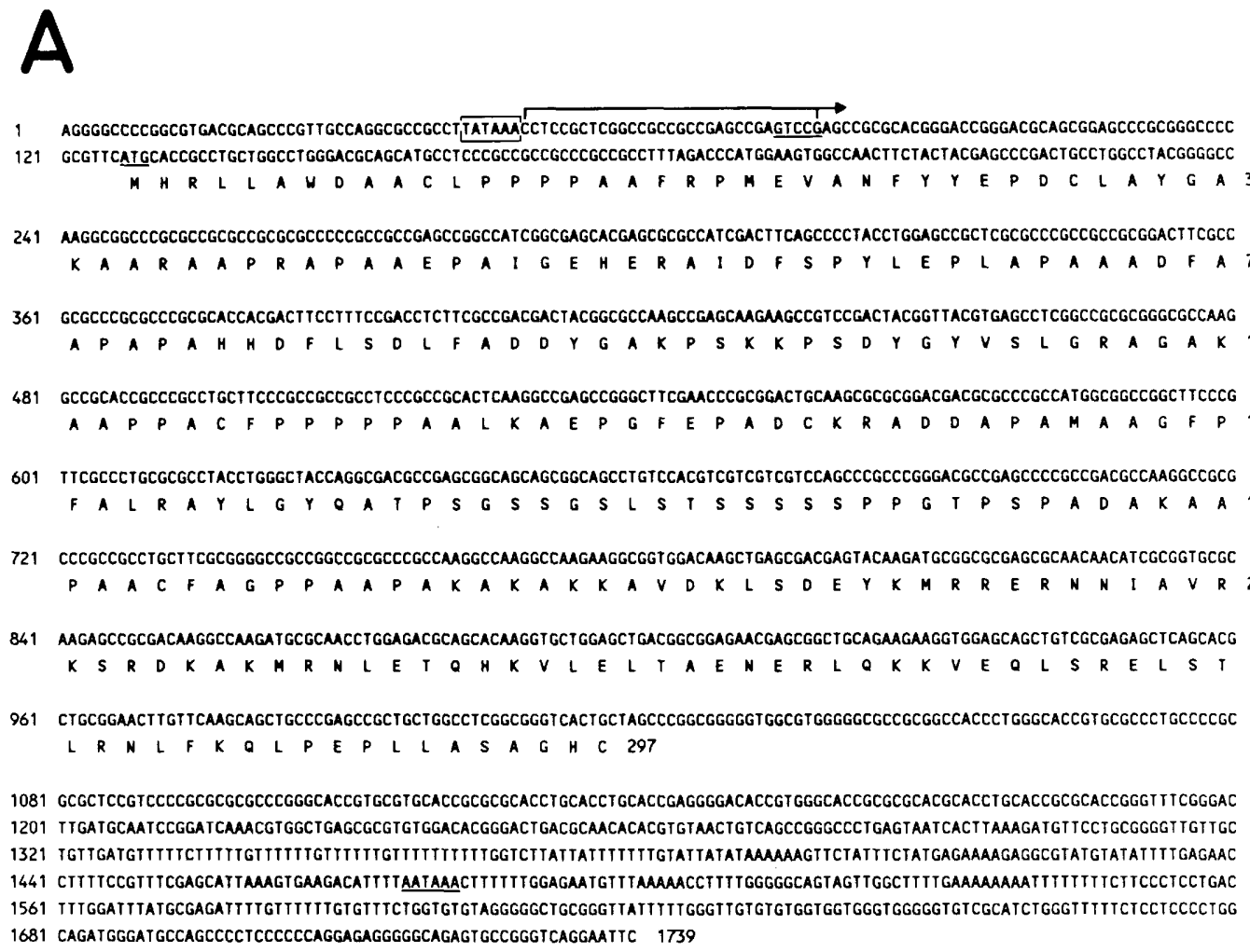
1681 CAGATGGGATGCCAGCCCCTCCCCCCAGGAGAGGGGGCAGAGTGCCGGGTCAGGAATTC 1739

B

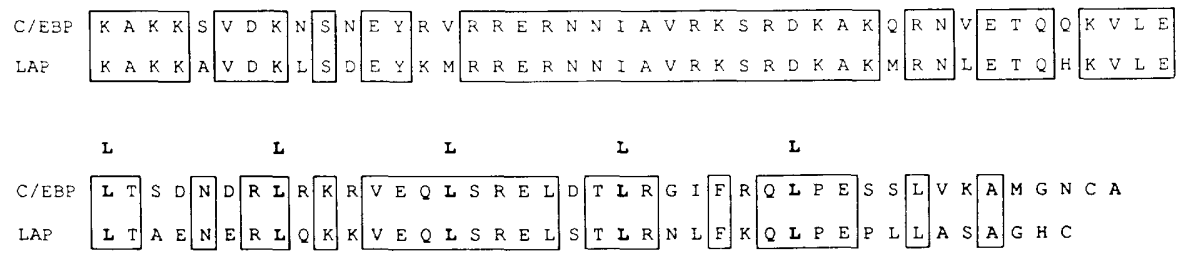

Figure 1. (A) Nucleotide sequence and deduced amino acid sequences of the LAP gene. The boxed nucleotides (42-47) represent the TATA box; the overlined sequence $(48-78)$ indicates the region containing the multiple transcriptional start sites. The major transcriptional start sites, as well as the ATG initiation codon, are underlined. The ORF starts at position 127 and ends at position 1017, encoding the 297-amino-acid LAP protein. The underlined sequence (1476-1481) indicates the polyadenylation signal; the poly(A) starts in the track of A between positions 1500 and 1504. (B) Comparison of the carboxy-terminal amino acid sequences of C/EBP and LAP spanning the basic domain and the leucine zipper. Sequence homology $(71 \%)$ is indicated by boxes.

generated at least as efficiently as the homodimeric forms of the two parent proteins. Because LAP and C/EBP coexist in liver, it is likely that these two proteins also heterodimerize in vivo (see Discussion).

\section{LAP is a major D-binding activity in rat liver nuclei}

In gel retardation experiments with the albumin $\mathrm{D}$ site as a probe, multiple DNA-protein complexes can be resolved (Lichtsteiner et al. 1987; Mueller et al. 1990). Recombinant cDNAs have been obtained for three of the proteins, DBP (Mueller et al. 1990), C/EBP (Landschulz et al. 1988), and LAP (this paper), interacting with this promoter element. An additional complex would be expected as a result of LAP-C/EBP heterodimer formation. To examine the contribution of LAP to the Dbinding activities, gel retardation experiments with liver nuclear extracts have been performed in the presence of polyclonal anti-LAP serum. This serum interacts with recombinant LAP and forms a complex with a slower migration on native polyacrylamide gel (supershift), whereas the preimmune serum does not interfere with complex formation (Fig. 3). Because the binding of LAP to the D-site oligonucleotide is not significantly inhibited in the presence of excess anti-LAP serum, the majority of antibodies present in this serum must decorate 
Descombes et al.
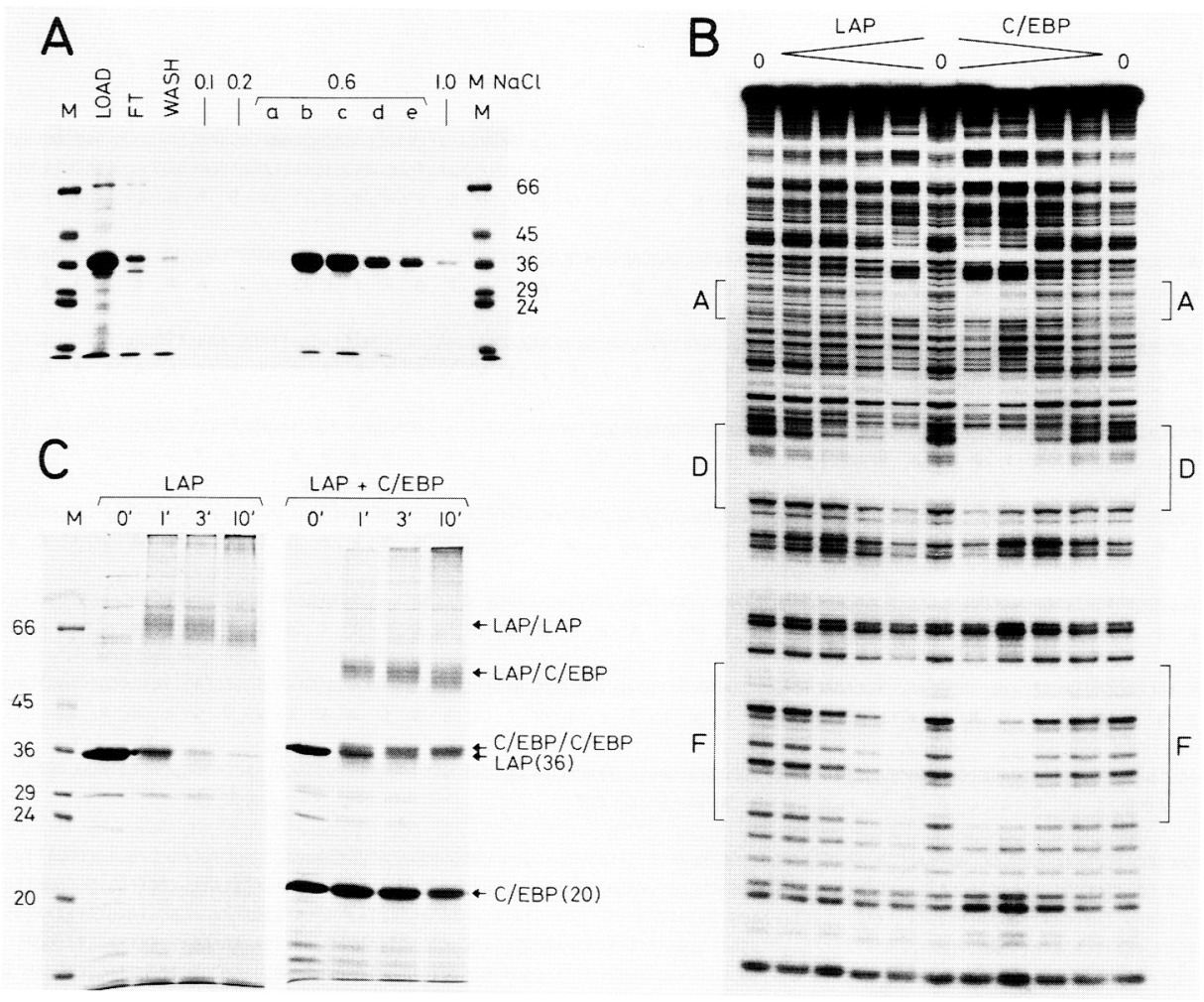

Figure 2. (A) Purification of recombinant LAP protein from Escherichia coli extracts by heparin-agarose chromatography. Equivalent portions of the bacterial extract (load) and the fractions eluting from the column were resolved by electrophoresis on a SDS-polyacrylamide gel and stained with Coomassie Blue. Samples are as follows: molecular weight markers (M), flowthrough (FT), wash, 0.1, $0.2,0.6(\mathrm{a}-\mathrm{e})$ and $1 \mathrm{M} \mathrm{NaCl} .(B)$ DNase I footprints obtained with LAP or C/EBP recombinant proteins on the albumin promoter. The locations of sites A, D, and F are indicated by brackets. Comparable footprinting units $(0,2,4,8$, and 12$)$ of LAP and C/EBP $(\mathrm{kindly}$ provided by S. McKnight) were used. (C) Dimerization of LAP with itself and with C/EBP. Recombinant LAP ( $36 \mathrm{kD}$ apparent molecular mass) was incubated alone (left) or with the carboxy-terminal moiety of C/EBP $(20 \mathrm{kD}$ apparent molecular mass) (right) in the presence of $0.01 \%$ glutaraldehyde (Landschulz et al. 1989) for 1, 3, and $10 \mathrm{~min}$. The complexes were resolved by electrophoresis on a $12 \%$ SDS-polyacrylamide gel. The positions of the LAP-LAP, LAP-C/EBP, and C/EBP-C/EBP dimers are shown by arrows.

epitopes that are not part of the DNA-binding/dimerization domain. Moreover C/EBP, whose sequence homology with LAP is limited to these domains, is not recognized by anti-LAP. With rat liver nuclear extracts, an appreciable fraction of the D-binding activities migrates more slowly in the presence of anti-LAP (Fig. 3). However, in contrast to the results obtained with recombinant LAP, anti-LAP also decreases the binding activity in rat nuclear extracts. The reason for this discrepancy is not known. Conceivably, post-translational modifications only occurring in eukaryotic cells, such as phosphorylations and/or glycosylations, augment the DNA binding affinity of LAP. Perhaps the peptide domains carrying such allosterically active groups become masked upon interactions with immunoglobulins. Irrespective of the mechanisms responsible for the somewhat different behavior of recombinant and rat liver LAP in the antibody interference experiments, these data suggest that at least $30 \%$ and possibly $70 \%$ of the rat liver D-binding activities can be attributed to LAP homodimers or heterodimers.

\section{LAP activates transcription in vivo and in vitro}

The transcription activation potential of LAP was examined in vivo by transient cotransfection experiments and in vitro by complementation of spleen nuclear extracts with recombinant LAP. An expression vector, pSCTLAP, suitable for LAP expression in mammalian cells, was constructed by inserting a restriction fragment containing most of the LAP ORF (amino acids 22-297) downstream of a cytomegalovirus enhancer/promoter (Rusconi et al. 1990). This expression vector was used in combination with the three reporter genes pAlb-CAT, $\left.\mathrm{pP}(\mathrm{D})\right|_{9}$-CAT, and $\left.\mathrm{pP}(\mathrm{E})\right)_{5}$-CAT (Fig. $\left.4 \mathrm{~A}\right)$ in cotransfection experiments with hepatoma HepG2 cells. The promoters of the reporter genes pAlb-CAT and $\mathrm{pP}(\mathrm{D})_{9}-$ CAT contain LAP recognition sequences, whereas $\mathrm{pP}(\mathrm{E})_{5}-\mathrm{CAT}$ has five albumin promoter elements $\mathrm{E}$ that are unable to interact with LAP. Cotransfection of pSCT-LAP with pAlb-CAT and pP $\left.(\mathrm{D})\right|_{9}-\mathrm{CAT}$ results in a 150- and 50-fold increase of CAT activity, respectively, as compared to the transfection of these two reporter 


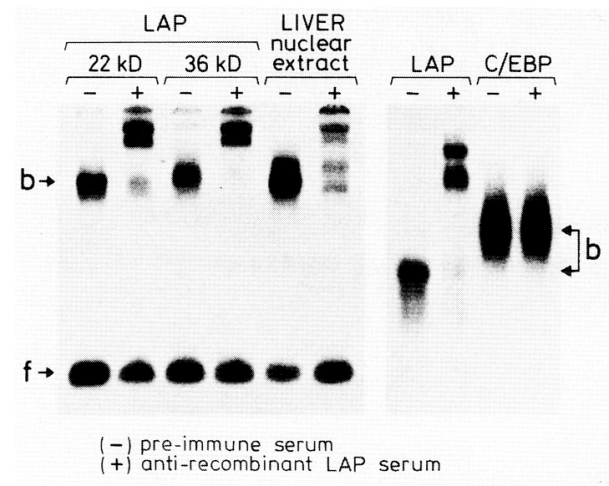

Figure 3. LAP D-binding activity in rat liver nuclei. (Left) Mobility-shift analysis of recombinant LAP proteins $(22$ - and 36-kD species) and liver nuclear extract; (right) mobility-shift analysis of recombinant LAP $(22 \mathrm{kD})$ and recombinant fulllength C/EBP proteins. Samples were incubated with either preimmune $(-)$ or anti-LAP $(+)$ serum and with a ${ }^{32} \mathrm{P}$-labeled site D oligonucleotide. The DNA-protein complexes were resolved by electrophoresis on a $6 \%$ nondenaturing polyacrylamide gel. The positions of free DNA $(\mathrm{f})$ and bound DNA $(\mathrm{b})$ are indicated. To increase the resolution between LAP-DNA and C/EBP-DNA complexes, the electrophoresis of the gel shown at right was carried out twice as long as the one shown at left. No complexes were observed in incubations containing LAP antiserum and DNA (data not shown).

genes alone. In contrast, no stimulation is observed in the cotransfection experiment with $\mathrm{pP}(\mathrm{E})_{5}-\mathrm{CAT}$, included as a negative control. These results suggest that LAP stimulates the transcription efficiency of the pAlb$\mathrm{CAT}$ and $\mathrm{pP}(\mathrm{D})_{9}-\mathrm{CAT}$ through interaction with its cognate sequences present in the promoters of these reporter genes. This conclusion is supported strongly by a complementation assay in a cell-free transcription system (Fig. 4B). Addition of purified recombinant LAP enhances in vitro transcription from a $P(D)_{9}$ fourfold in spleen nuclear extracts containing low levels of site Dbinding activities. At very high LAP concentrations, the transcriptional activation is diminished. Perhaps, excessive amounts of this activator protein may bind in solution to a critical component of the general transcription apparatus, thereby competing for its protein surface required for the interaction of LAP bound to its DNA recognition sequence. This "squelching" phenomenon has been observed with other strong activator proteins (Ptashne 1988). In accordance with the results obtained in cotransfection experiments, LAP requires a functional recognition element to stimulate in vitro transcription, because the activity of the adenovirus major late promoter, included as an internal control in the in vitro complementation assays, is not affected by the addition of recombinant LAP.

\section{The expression of LAP in different rat tissues}

As reported earlier (Lichtsteiner et al. 1987), liver nuclei contain considerably higher levels of D-binding factors than nuclei from spleen or brain. Because LAP may well be the major D-binding rat liver factor (see above), it should also be more concentrated in liver nuclei than in nuclei of other tissues. This speculation was tested by a Western blot experiment. Nuclei were purified from liver, spleen, brain, testis, lung, and kidney. After sonication of the nuclei, total nuclear proteins were solubilized directly by the addition of SDS-loading buffer (Gasser et al. 1986). This procedure ensures complete recovery of histone and nonhistone proteins, unlike the conventional salt extraction methods commonly used for the purification of nuclear proteins. As seen in Figure $5 \mathrm{~A}$, the immunochemical analysis of electrophoretically separated proteins clearly reveals a strong signal corresponding to a protein that comigrates with recombinant LAP (apparent molecular mass, $36 \mathrm{kD}$ ) in the liver nuclear lysate. LAP is also detected in lung nuclei, albeit at a level $\sim 5$ - to 10 -fold lower. Nuclear lysates from the remaining four tissues, spleen, brain, testis, and kidney, as well as cytoplasmic extracts from lung and liver, appear to contain little, if any, LAP.

In the liver nuclear lysate, a protein band with a slower migration reacts with anti-LAP in addition to the $36-\mathrm{kD}$ LAP species. The ratio of the radioactive signals associated with the upper and lower protein species is

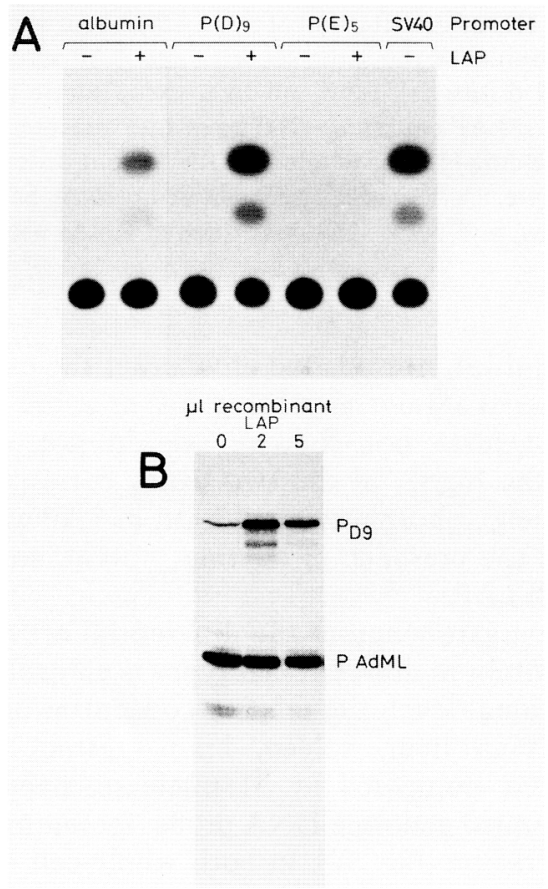

Figure 4. LAP activates transcription in vivo and in vitro. $(A)$ CAT activities of extracts from hepatoma HepG2 cells transfected with $(+)$ or without $(-)$ an expression vector encoding LAP (pSCT-LAP, amino acids 22-297) and a CAT-reporter gene containing one of the following promoters: mouse albumin promoter (albumin), a synthetic promoter consisting of nine $\mathrm{D}$ elements $\left[\mathrm{P}(\mathrm{D})_{9}\right]$, and a synthetic promoter consisting of five E elements $\left[\mathrm{P}(\mathrm{E})_{5}\right]$. To monitor the efficiency of transfection, cells were also transfected with a CAT-reporter gene containing the promoter of the SV40 early transcription unit (SV40). 


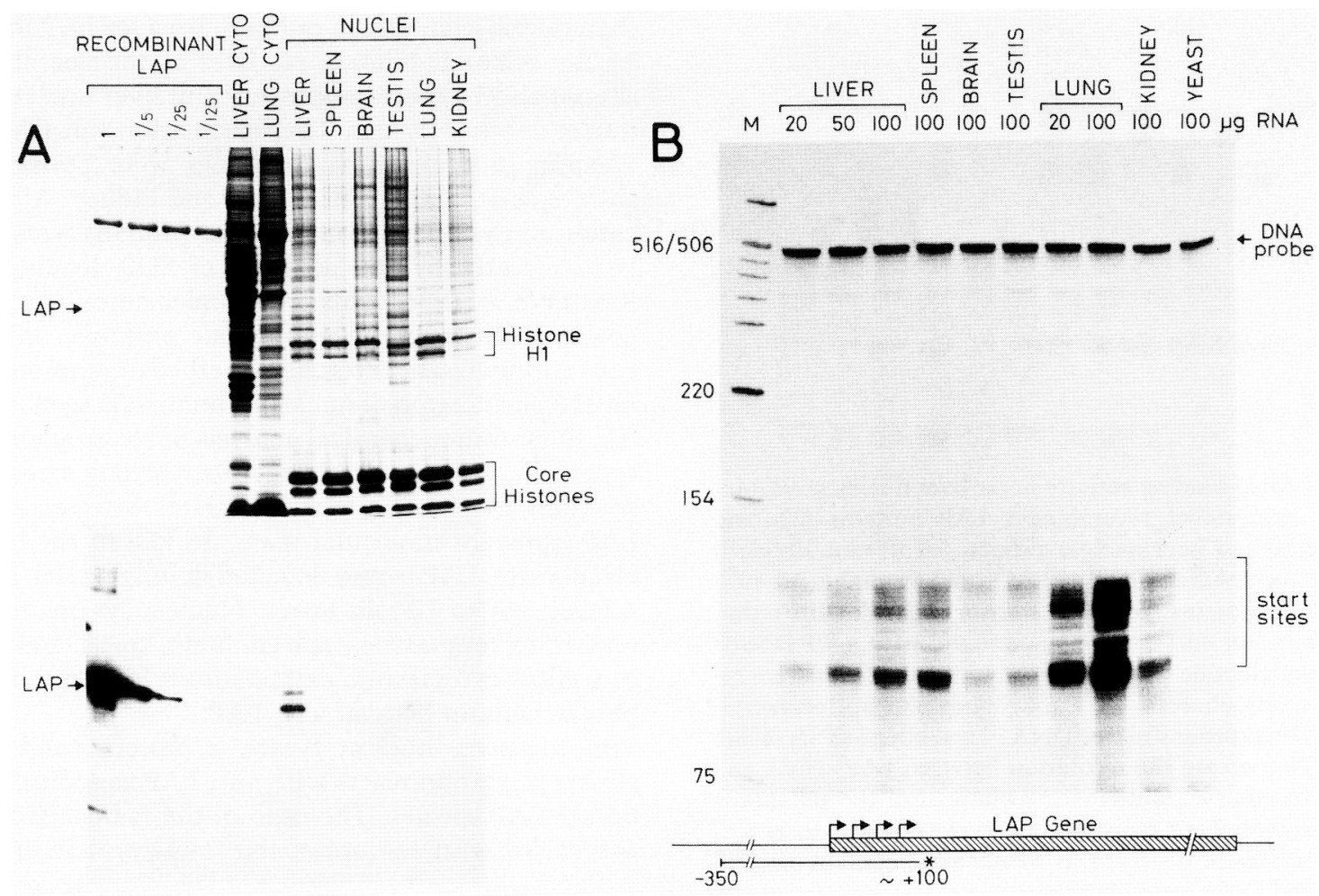

Figure 5. Tissue distribution of the LAP protein and putative LAP mRNA. (A) Coomassie Blue-stained SDS-polyacrylamide gel $(t o p)$ and immunoblot of the same gel with anti-LAP serum (bottom). Samples are as follows: recombinant LAP protein $(1: 1,1: 5,1: 25$, and $1: 125$ dilutions $\mid$, lung and liver cytoplasmic proteins, and nuclear proteins from the indicated tissues. The $1: 1$ dilution of recombinant LAP contains $\sim 100 \mathrm{ng}$ of LAP protein. Four micrograms of BSA were included in each sample of recombinant LAP. $(B)$ S1 nuclease mapping of whole-cell RNA from different tissues by using a single-stranded genomic DNA probe, including the LAP gene promoter (schematically shown at bottom). Samples are as follows: molecular weight markers (M), liver, spleen, brain, testis, lung, kidney, and yeast. The quantities of RNAs used in the Sl nuclease mapping are given in micrograms.

about one-third, irrespective of whether the nuclei have been prepared at neutral $\mathrm{pH}$ (Fig. $5 \mathrm{~A}$ ) or at $\mathrm{pH} 1.7$ in $5 \%$ citric acid (data not shown). We do not yet know whether the higher of the two bands corresponds to a modified (e.g., phosphorylated) form of LAP or whether it reflects the presence of another protein, sharing epitopes with LAP.

To investigate whether the differential accumulation of LAP protein in different cell types is controlled at the transcriptional level, the relative concentrations of LAP transcripts in liver, spleen, brain, testis, lung, and kidney were compared by S1 nuclease mapping with single-stranded genomic DNA probe, including the LAP promoter region (Fig. 5B). In both whole-cell RNA (Fig. 5B) and cytoplasmic RNA (data not shown) preparations, correctly initiated LAP transcripts can be detected in all of the analyzed tissues. Unexpectedly, the highest level of putative LAP mRNA is observed in lung. This transcript is $\sim 5$ - to 10-fold less abundant in liver, spleen, and kidney and even sparser in brain and testis. Virtually identical results have been obtained with a DNA probe spanning the entire ORF (data not shown). On the basis of screening of the oligo(dT)-primed cDNA library with the original LAP cDNA insert as a hybridization probe, we can estimate the relative abundancy of LAP mRNA in liver, and by extrapolation, in other tissues. The screening of $5.5 \times 10^{5}$ recombinant plaques yielded 133 positives, corresponding to a relative frequency of $2.4 \times 10^{-4}$. On the basis of the S1 mapping data presented in Figure 5B this value is expected to be $\sim 10$-fold higher in lung, suggesting that the relative LAP mRNA concentration in this tissue may approach $0.2 \%$ of the cellular mRNA.

Comparison of Figure 5, A and B, shows that the relative LAP protein and RNA levels do not correlate in four (liver, lung, spleen, and kidney) of the six tissues analyzed. The discrepancy between LAP mRNA and protein abundance is particularly striking in liver and lung. As judged from our Western blot and S1 nuclease mapping experiments, the LAP protein/mRNA ratio is $\sim 50$ - to 100 -fold higher in liver than in lung. As a result of the nuclear localization of LAP (Fig. 5A), this ratio should be corrected for eventual differences in cytoplasmic nuclear proportions in the expressing cell types. Although we did not yet identify these cell types positively, this correction is not anticipated to account for the large discrepancy of the LAP protein/mRNA ratio in these two tissues. More likely, these data reflect a post-transcriptional modulation of LAP expression in different cell types. 


\section{Discussion}

We have isolated and characterized a gene specifying a transcriptional activator protein, LAP, that binds to the albumin promoter element $D$. This gene, like the one specifying C/EBP (Landschulz et al. 1988), is not interrupted by intervening sequences. Varying amounts of putative mRNA are present in the six tissues that we investigated. Putative LAP mRNA levels are highest in lung, intermediate in liver, spleen, and kidney, and lowest in brain and testis. In contrast to the tissue distribution of LAP transcripts, LAP protein accumulates to a much higher concentration in liver than in lung nuclei and is barely detectable in the other four tissues. The discrepancy between protein and mRNA levels observed in the various tissues could be due to (1) largely different cytoplasmic to nuclear ratios in different cell types, (2) preferential translatability of LAP mRNA in the expressing tissue, and (3) differential stability of LAP in different cell types. The first possibility could not be addressed because we have not yet identified the cell types accumulating LAP mRNA and protein by in situ hybridization and immunohistochemistry, respectively. Given the large difference of the ratio of LAP protein to LAP mRNA in different tissues, we favor the second or the third possibility. What, then, is the significance, if any, of LAP mRNA in tissues that do not constitutively produce LAP? Perhaps, under certain physiological conditions, the accumulation of LAP may be inducible via a translational or post-translational mechanism in nonhepatic cells.

LAP is the third D-binding rat liver protein for which recombinant DNAs have been obtained and whose sequence has been determined. It shares extensive sequence similarity with the DNA-binding and dimerization domains of the CCAAT/enhancer-binding protein C/EBP (Landschulz et al. 1989). This sequence homology is functionally relevant, because the two proteins manifest the same DNA sequence specificity, at least with regard to the albumin promoter elements, and can heterodimerize. Despite their divergent amino-terminal regions, both LAP (Fig. 4A) and C/EBP (Friedman et al. 1989; P. Maire and U. Schibler, unpubl.) strongly enhance transcription from the albumin promoter or from a synthetic promoter containing multiple cognate sites for these two proteins. In similar experiments the same transcriptional activation has been observed for DBP (Mueller et al. 1990). Thus, at least three proteins, as well as a possible fourth, consisting of heterodimers of C/EBP and LAP, can potentially perform the same function via the same cis-acting element.

Whether the functional redundancy observed in transient cotransfection experiments (schematically illustrated in Fig. 6A) applies to the multiple D-binding factors in their natural environment, the hepatocytes, can not be answered presently. Many important parameters, such as the ratio of active transcription factors to accessible target sequences or the precise chromatin structure of a given gene, are not reproduced in these simple assay systems. Therefore, one may also consider that transcription factors with similar in vitro binding

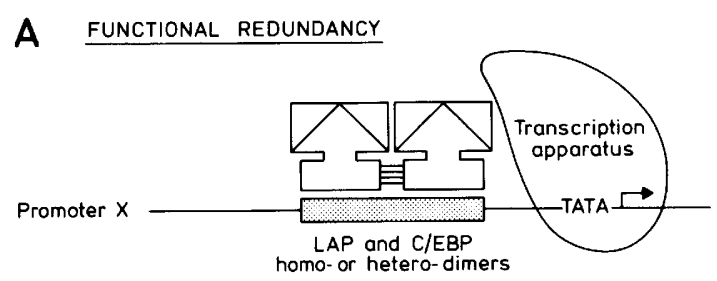

B DISTINCT FUNCTIONS
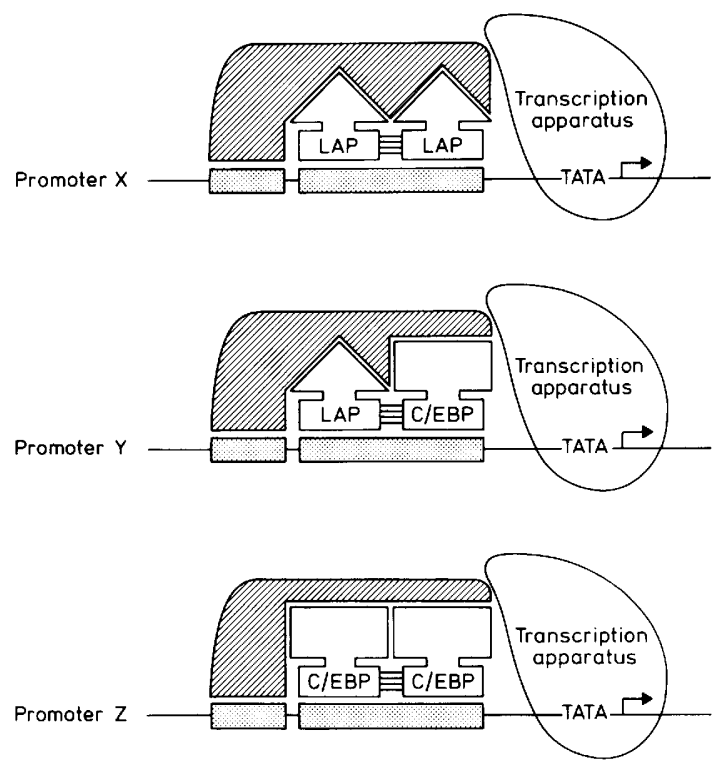

Figure 6. Two alternative models representing either identical or distinct functions for LAP and C/EBP homo- and heterodimers. (A) Functional redundancy. Homo- and heterodimers of LAP and C/EBP bind to the same cis-acting elements and augment the transcription frequency by contacting a surface of the general transcription apparatus (e.g., RNA polymerase II, TFIID, etc.). (B) Distinct functions. In this model, homo- and heterodimers of LAP and C/EBP bind to cis-acting elements of different target genes. For simplicity, the specific cooperative interaction with a single additional factor is shown. Specificity of binding may also be accomplished by cooperative interactions with multiple factors.

specificities might perform distinct functions within their natural context. A simple version of such a model is illustrated for C/EBP and LAP in Figure 6B. Conceivably, the homo- and heterodimers between LAP and C/EBP are guided to promoters (or enhancers) of different genes by virtue of cooperative interactions with other transcription factors. According to this scheme, the divergent amino-terminal domains of LAP and $\mathrm{C} / \mathrm{EBP}$ would have to interact specifically with distinct surfaces of different factors bound to nearby cis-acting elements to form stable preinitiation complexes. This model would have an interesting corollary: A change in the concentration of a single component, LAP or C/EBP, would differentially affect the expression of the genes controlled by LAP homodimers, LAP-C/EBP hetero- 
dimers, and C/EBP homodimers. For example, a significant increase in the level of LAP alone would result in a substantial augmentation of LAP homodimers, a moderate increase of LAP-C/EBP heterodimers, and a marked decrease of C/EBP homodimers. As a consequence, in the model shown in Figure 6B, transcription from promoter $X$ would be stimulated strongly, transcription from promoter $Y$ would be enhanced slightly, and transcription from promoter $Z$ would be reduced markedly. A similar model has been discussed recently for the two related oncogenes c-Jun and Jun-B (Schütte et al. 1989). These two proteins have distinct properties, probably specified by their different activation domains (Chiu et al. 1989). c-Jun, but not Jun-B, can activate transcription of the c-iun gene. The concomittant expression of Jun-B and c-Jun results in the inhibition of c-Jun action, conceivably by sequestering c-Jun into a heterodimer with Jun-B that is less active than C-Jun homodimers (or c-Jun-c-Fos heterodimers). Homo- and heterodimers can also be formed between the two Drosophila AP-1-related proteins dFRA and dJRA (Perkins et al. 1990) and the multiple ATF-related factors (Hai et al. 1989). A second class of heterodimeric transcriptional activators, exemplified by the mammalian Fos-Jun complexes (for references, see Zerial et al. 1989|, is composed of one protein that can bind DNA sequences autonomously (i.e., Jun) and another that cannot (i.e., Fos). In yet a third class of heterodimeric factors, both subunits are required for DNA binding. An example of such a protein complex is the pancreas-specific transcription factor PTF1 (Roux et al. 1989).

The binding of multiple distinct factors to the same DNA motifs has been reported in a variety of systems. A well-documented example is the binding of alternative transcription factors to a conserved octamer sequence present in the promoter regions of several genes, including the ones encoding immunoglobulins, histones, herpes simplex virus proteins, and small nuclear RNAs. OTF-1 appears to be present in most cell types, whereas OTF-2 is a B-cell specific factor (for review, see Schaffner 1989). As in the case of LAP and C/EBP, octamer-binding factors recognize the same DNA elements through a conserved DNA-binding domain. According to a recently published report and in contrast to the three distinct D-binding proteins LAP, DBP, and C/EBP, OTF- 1 and OTF-2 perform different functions and cannot be substituted for each other in transient cotransfection assays (Tanaka and Herr 1990). OTF-2, in contrast to OTF-1, relies on two interdependent nonacidic protein domains for its activity. These two activation domains are required for the characteristic phosphorylation pattern of OTF-2 (Tanaka and Herr 1990). We do not yet know to what extent similar post-translational modifications are involved in modulating the activity of LAP and other D-binding factors. Like CREB (Gonzalez and Montminy 1989), LAP has a characteristic phosphoacceptor sequence for CAMP-dependent protein kinase in its amino-terminal region and undergoes phosphorylation in vitro with the catalytic subunit of protein kinase A (M. Chojkier, unpubl.). Perhaps, phosphorylation of
LAP may affect its interaction with specific DNA sequences and/or other transcription factors. This hypothesis can now be assessed by searching for nuclear proteins and/or DNA recognition sequences that may interact specifically with unphosphorylated and in vitro-phosphorylated forms of recombinant LAP.

\section{Materials and methods}

Animals, cells, and materials

Three- to four-year-old male rats of the inbred strain Lewis were used. HepG2 hepatoma cells (kindly provided by P. Maire, INSERM, Paris) were grown as monolayers in minimal essential medium supplemented with $5 \%$ fetal calf serum. A plasmid, pMSV-C/EBP, containing C/EBP cDNA insert and a bacterial extract containing full-length recombinant C/EBP were generously provided by S. McKnight /Carnegie Institution of Washington, Baltimore).

\section{Cloning of the LAP CDNA and the LAP gene}

A recombinant liver cDNA $\lambda g t 11$ expression library was constructed as described previously (Mueller et al. 1990), except that random hexameric oligonucleotides instead of dT oligomers were used as primers for the synthesis of the first cDNA strand. Over $95 \%$ of the $7 \times 10^{6}$ phage plaques obtained from 1 $\mu \mathrm{g}$ of in vitro-packaged recombinant DNA contained cDNA inserts (average molecular mass, $1 \mathrm{kD}$ ). Ten nitrocellulose filters containing a total of $2 \times 10^{5}$ recombinant plaques were screened according to Singh et al. (1988) and Vinson et al. (1988) by use of an oligomerized, double-stranded mutant 10 oligonucleotide (TGGTATGATGTTGTAATGGGG; underlined G is T in the wild-type sequence). Four plaques were positive in both screening procedures, and their recombinant phages were purified. The fusion proteins encoded by three of these phages, when tested in a binding assay with different radiolabeled oligonucleotides, behaved like purified rat liver D-binding proteins. They show a high affinity for the wild-type and mutant 10 oligonucleotides, have a very weak affinity for mutant 4 oligonucleotide (TGGTATGATTTTGTAC̣TGGGG; underlined $\mathrm{C}$ is $\mathrm{A}$ in the wild-type sequence), and no measurable affinity for an unrelated oligonucleotide, the albumin promoter element B (Lichtsteiner and Schibler 1989). The insert of one of these partial LAP cDNA clones was used to screen an amplified oligo(dT)-primed $\lambda g t 11$ library (Mueller et al. 1990) and a genomic EMBL-3 rat DNA library (generous gift of A. Kahn) by hybridization (Sambrook et al. 1989). The longest cDNA insert (1438 bp) thus obtained was subcloned into a plasmid (pBS +) and sequenced (Sanger et al. 1977; Maxam and Gilbert 1980). Two overlapping genomic clones, which together span $\sim 17 \mathrm{~kb}$, were characterized by digestion with several restriction endonucleases. A 2.3-kb BamHI-EcoRI fragment hybridizing in Southern blot experiments to the LAP cDNA was subcloned into $\mathrm{pBS}+$, and the nucleotide sequence of the DNA region containing the LAP gene was determined (Sanger et al. 1977; Maxam and Gilbert 1980).

\section{Overexpression and purification of recombinant proteins}

A partial $\mathrm{NcoI} / S m a$ I DNA fragment containing the LAP ORF (between positions 189 and 1103) was inserted into pET 8c (Studier et al. 1989), which had been digested previously with $\mathrm{NcOI}$ and BamHI (blunted). The resulting chimeric plasmid was used to transform the bacterial strain BL 21/DE-3/pLysS (Studier et al. 1990). This plasmid encodes a LAP protein 
lacking only the amino-terminal 21 amino acids. An expression plasmid encoding a short version of LAP was constructed by inserting a blunted $E c o$ RI fragment of one of the partial cDNAs obtained in the original screening into the blunted $\mathrm{BamHI}$ site of the phage T7 expression vector pET $3 b$ (Studier et al. 1990). The fusion protein produced from this vector contains 15 amino acids encoded by the $\mathrm{T} 7$ vector and the carboxy-terminal 177 amino acids of LAP. An expression vector encoding a truncated version of C/EBP (carboxy-terminal 143 amino acids) was obtained by cloning a blunted PstI fragment of the C/EBP cDNA into the blunted BamHI site of pET 3a (Studier et al. 1990).

Bacterial extracts were prepared from bacteria (BL 21/DE-3/ pLysS/grown for 4-5 hr in the presence of $0.5 \mathrm{~mm}$ IPTG, as described by Landschulz et al. (1989). Recombinant proteins were purified from these lysates by fractionation on heparinagarose columns as was described previously (Lichtsteiner et al. 1987), except that the load and the wash contained $5 \mathrm{M}$ urea (see Fig. 2).

\section{Western blot analysis of nuclear and cytoplasmic proteins}

Tissues ( 3 grams of each) were obtained from adult male rats. Nuclei were prepared in homogenization buffer containing $1 \%$ (wt/vol) low-fat dry milk (which, in our laboratory, has been shown to be a potent competitive protease inhibitor) and resuspended in $2 \mathrm{ml}$ ( $6 \mathrm{ml}$ for spleen) of lysis buffer, as decribed previously (Lichtsteiner and Schibler 1989; Maire et al. 1989). The nuclear suspensions were sonicated extensively on ice, mixed with $2 \times$ SDS-loading buffer (Laemmli 1970), heated at $90^{\circ} \mathrm{C}$ for $5 \mathrm{~min}$, and resonicated. For cytoplasmic protein preparations, 1.3 grams of lung and 2.2 grams of liver were homogenized extensively in $12 \mathrm{ml}$ of homogenization buffer as above but with only $0.5 \mathrm{M}$ sucrose and without low-fat milk. The homogenates were centrifuged for $10 \mathrm{~min}$ at $3000 \mathrm{rpm}$ in a Sorvall HB-4 rotor to remove the nuclei. Aliquots of the supernatants were mixed with equal volumes of $2 \times$ SDS loading buffer. Equivalents of $0.4 \mathrm{OD}_{260}$ units of nuclear lysates and $30 \mu \mathrm{l}$ of cytoplasmic extracts in $1 \times$ SDS loading buffer were electrophoresed under reducing conditions on a $12 \%$ SDS-polyacrylamide gel (Laemmli 1970), along with full-length recombinant LAP protein and molecular weight standards. The gel was stained with Coomassie Blue, destained, and transferred onto nitrocellulose (Harlow and Lane 1988). LAP was detected by autoradiography following incubation of the nitrocellulose membrane with rabbit polyclonal anti-LAP serum $(1: 200$ dilution $)$ and 125I-labeled protein A $(0.2 \mu \mathrm{Ci} / \mathrm{ml}$, Amersham). Rabbit LAP an tibodies were raised according to standard procedures (Harlow and Lane 1988) against a purified fusion protein containing the carboxy-terminal 177 amino acids of LAP and the amino-terminal 15 amino acids encoded by the phage T7 expression vector pET 3b (Studier et al. 1990). Briefly, $\sim 1 \mathrm{mg}$ of recombinant LAP protein, purified by heparin-agarose chromatography, was mixed with complete Freund's adjuvant and injected subcutaneously into a rabbit. Two boosts with the same quantity of recombinant LAP, but mixed with incomplete Freund's adjuvant, were given after 3 and 6 weeks, respectively. Blood was collected 10 weeks after the initial immunization.

\section{Transfection experiments}

Transfection, preparation of cellular extracts, determination of the CAT activity, and thin layer chromatography were performed as described previously (Mueller et al. 1990). The CAT plasmids containing the albumin and the $(D)_{9}$ promoter have been described elsewhere (Mueller et al. 1990). The CAT plasmid containing five $\mathrm{E}$ sites has been provided generously by P. Maire (INSERM, Paris). The LAP expression vector (pSCTLAP) was obtained starting with pMSV-C/EBP (generous gift of S. McKnight): a NcoI-HindIII fragment of 510 bp of pMSV-C/ EBP [containing the murine sarcoma virus (MSV) LTR and the ATG consensus sequence] was isolated and ligated to the LAP gene (in pBS + ) cut with HindIII and $\mathrm{NcOI}$ (excludes nucleotides 1-676). The resulting plasmid (pMSV-LAP) was digested completely with BamHI and partially with SmaI. The 910-bp fragment (containing the ATG consensus sequence from pSCT $-\mathrm{C}$ / EBP and the entire ORF of LAP) was inserted into pSCT Gal X556 (generous gift of S. Rusconi) cut with BamHI and PvuII, resulting in pSCT-LAP, in which the polyadenylation signal comes from the $\beta$-globin gene. In this vector, the transcription of a LAP mRNA is driven by the cytomegalovinus enhancer/ promoter (Rusconi et al. 1990). An RSV-luciferase plasmid (2 $\mu \mathrm{g} /$ plate) was included in the transfection experiments to correct for variability in the transfection efficiency (De Wet et al. 1987).

\section{S1 nuclease mapping}

Whole-cell RNAs were prepared from 2 grams of each tissue (liver, spleen, brain, testis, lung, and kidney), according to the method described by Clemens et al. (1984). For S1 nuclease mapping experiments a quasi-end-labeled single-stranded DNA (ssDNA) probe was prepared as follows. ssDNA of the LAPcoding strand (mRNA sequence) was prepared according to Sambrook et al. (1989). Bacteria (JM109) were transformed with a pBS + plasmid containing a genomic BamHI-EcoRI LAP fragment. Exponentially growing bacterial cells were infected with the helper phage M13KO7. ssDNA was recovered from phenolextracted phage particles and used as a template for a primer extension reaction with the oligonucleotide primer $5^{\prime}$ CGGCGGCGGGAGGCATGCTGCGTCCC-3'. The primer extension cocktail $(50 \mu \mathrm{l})$ contained $500 \mathrm{ng}$ of single-stranded template DNA, $50 \mathrm{ng}$ of primer, $1 \times$ Sequenase buffer (U.S. Biochemical Corporation, Sequenase kit), $0.15 \mathrm{M} \mathrm{NaCl}$ (in addition to the Sequenase buffer), $200 \mu \mathrm{M}$ each of dATP, dGTP, dTTP, 50 $\mu \mathrm{Ci}$ of $\left[\alpha^{-32}\right] \mathrm{dCTP}, 20$ units of T7 DNA polymerase (U.S. Biochemical Corporation, Sequenase kit), and 20 units of the restriction endonuclease $\mathrm{BamHI}$. After $15 \mathrm{~min}$ at $37^{\circ} \mathrm{C}, 1 \mu \mathrm{l}$ of 20 mM dCTP was added to the reaction, and the incubation was continued for another $15 \mathrm{~min}$. The DNA was phenol-extracted, precipitated with ethanol, and resolved on a $4 \%$ urea-polyacrylamide gel. After exposure of the wet gel for $10 \mathrm{~min}$ at room temperature, the ssDNA band (spanning the LAP promoter) corresponding to $\sim 500$ nucleotides, was eluted from the polyacrylamide by soaking of the gel slice for $3 \mathrm{hr}$ at room temperature in $2.4 \mathrm{ml}$ of $0.5 \mathrm{M}$ sodium acetate, $1 \mathrm{mM}$ EDTA, and 100 $\mu \mathrm{g} / \mathrm{ml}$ single-stranded salmon sperm DNA. The DNA probe was recovered from the supernatant and concentrated by ethanol precipitation. $S 1$ nuclease mapping with the indicated amounts of RNAs and $10^{5} \mathrm{cpm}$ of the ssDNA probe was carried out as decribed by Sierra et al. (1990). S1-nuclease resistant DNA fragments were resolved on a $6 \%$ urea-polyacrylamide gel.

\section{DNase protection and gel retardation experiments}

The preparation of an end-labeled albumin promoter DNA fragment, as well as the DNase I protection analysis, were carried out as described (Lichtsteiner et al. 1987). Gel retardation analysis of protein-DNA complexes were performed as with an oligonucleotide spanning site D of the albumin promoter, as described earlier (Mueller et al. 1990). For the gel retardation ex- 
periments in the presence of anti-LAP serum (Fig. 3), recombinant LAP (22- and $36-\mathrm{kD}$ species), liver nuclear extract (Lichtsteiner et al. 1987), and recombinant C/EBP (generous gift of $\mathrm{S}$. McKnight) were diluted to yield protein concentrations that bound at least $50 \%$ of the added DNA. Before the addition of radiolabeled DNA, the protein samples were incubated with a 100 -fold dilution of either preimmune or anti-LAP serum for 2 hr at $25^{\circ} \mathrm{C}$.

\section{Other techniques}

Conventional recombinant DNA procedures were performed according to Sambrook et al. (1989). In vitro transcriptions were conducted as described by Lichtsteiner and Schibler (1989), with minor modifications: The $20-\mu \mathrm{l}$ reactions contained $40 \mu \mathrm{g}$ of spleen nuclear extract, $800 \mathrm{ng}$ of a G-free cassette plasmid bearing the $P(D)_{9}$ promoter, and $200 \mathrm{ng}$ of a G-free cassette plasmid bearing the adenovirus major late promoter. Recombinant LAP protein was included in some of the reactions (see legend to Fig. 4). Dimerization of proteins was examined according to Landschulz et al. (1989).

\section{Acknowledgments}

We thank S. McKnight for his generous gifts of C/EBP recombinant protein and DNA, W. Studier for the phage T7 expression system, P. Maire for several CAT reporter gene plasmids and for his advice in the transfection experiments, A. Kahn for a EMBL-3 rat DNA recombinant library, F. Fleury and C.H. Tonka for excellent technical assistance, $O$. Jenni for preparing the art work, and J. Bouverat for his help in preparing rabbit antisera. This work was supported by the Swiss National Science Foundation, the Zyma Foundation, the University of California, the National Institutes of Health, the Veterans Administration, and the State of Geneva.

The publication costs of this article were defrayed in part by payment of page charges. This article must therefore be hereby marked "advertisement" in accordance with 18 USC section 1734 solely to indicate this fact.

\section{Note added in proof}

Sequence data described in this paper have been submitted to EMBL/GenBank Data Libraries.

\section{References}

Birkenmeier, E.H., B. Gwynn, S. Howard, J. Jerry, J.I. Gordon, W.H. Landschulz, and S.L. McKnight. 1989. Tissue-specific expression, developmental regulation, and genetic mapping of the gene encoding CCAAT/enhancer binding protein. Genes Dev. 3: 1146-1156.

Cereghini, S., M. Blumenfeld, and M. Yaniv. 1988. A liver-specific factor essential for albumin transcription differs between differentiated and dedifferentiated rat hepatoma cells. Genes Dev. 2: 957-974.

Chiu, R., P. Angel, and M. Karin. 1989. Jun-B differs in its biological properties from, and is a negative regulator of, $\mathrm{c}$-Jun. Cell 59: 979-986.

Clemens, M.J. 1984. Purification of eukaryotic messenger RNA. In Transcription and translation: a practical approach. (ed. B.D. James and S.J. Higgins.) pp. 211-230. IRL Press, Oxford.

Courtois, G., S. Baumhueter, and G.R. Crabtree. 1988. Purified hepatocyte nuclear factor 1 interacts with a family of hepatocyte-specific promoters. Proc. Natl. Acad. Sci. 85: 79377941.
De Wet, J.R., K.V. Wood, M. DeLuca, D.R. Helinski, and S. Subramani. 1987. Firefly luciferase gene: Structure and expression in mammalian cells. Mol. Cell. Biol. 7: 725-737.

Frain, M., G. Swart, P. Monaci, A. Nicosia, S. Stämpfli, R. Frank, and R. Cortese. 1989. The liver-specific transcription factor LF-B1 contains a highly diverged homeobox DNA binding domain. Cell 59: 145-157.

Friedman, A.D., W.H. Landschulz, and S.L. McKnight. 1989. C/EBP activates the promoter of the serum albumin gene in cultured hepatoma cells. Genes Dev. 3: 1314-1322.

Gasser S.M., T. Laroche, J. Falquet, E. Boy de la Tour, and U.K. Laemmli. 1986. Metaphase chromosome structure: involvement of topoisomerase II. J. Mol. Biol. 188: 613-629.

Gonzalez, G.A. and M.R. Montminy. 1989. Cyclic AMP stimulates somatostatin gene transcription by phosphorylation of CREB at serin 133. Cell 59: 675-680.

Gorski, K., M. Carneiro, and U. Schibler. 1986. Tissue-specific in vitro transcription from the mouse albumin promoter. Cell 47: 767-776.

Hai, T., F. Liu, W.J. Coukos, and M.R. Green. 1989. Transcription factor ATF cDNA clones: An extensive family of leucine zipper proteins able to selectively form DNA-binding heterodimers. Genes Dev. 3: 2083-2090.

Harlow, E. and D. Lane. 1988. Antibodies: A laboratory manual. Cold Spring Harbor Laboratory Press, Cold Spring Harbor, New York.

Heard, J.M., P. Herbomel, M.O. Ott, A. Mottura-Rollier, M. Weiss, and M. Yaniv. 1987. Determinants of rat albumin promoter tissue specificity analyzed by an improved transient expression system. Mol. Cell. Biol. 7: 2425-2434.

Laemmli, U.K. 1970. Cleavage of structural proteins during the assembly of the head of bacteriophage T4. Nature 227: 680685.

Landschulz, W.H., P.F. Johnson, E.Y. Adashi, B.J. Graves, and S.L. McKnight. 1988. Isolation of a recombinant copy of the gene encoding C/EBP. Genes Dev. 2: 786-800.

Landschulz, W.H., P.F. Johnson, and S.L. McKnight. 1989. The DNA binding domain of the rat liver nuclear protein C/EBP is bipartite. Science 243: $1681-1688$

Lichtsteiner, S. and U. Schibler. 1989. A glycosylated liver-specific transcription factor stimulates transcription of the albumin gene. Cell 57: 1179-1187.

Lichtsteiner, S., J. Wuarin, and U. Schibler. 1987. The interplay of DNA-binding proteins on the promoter of the mouse albumin gene. Cell 51: 963-973.

Maire, P., J. Wuarin, and U. Schibler. 1989. The role of cisacting elements in tissue-specific albumin gene transcription. Science 244: 343-346.

Maxam, A.M. and W. Gilbert. 1980. Sequencing end-labeled DNA with base-specific chemical cleavages. Methods Enzymol. 65: 499-670.

Mueller, C.R., P. Maire, and U. Schibler. 1990. DBP, a liverenriched transcriptional activator is expressed late in ontogeny and its tissue-specificity is determined post-transcriptionally. Cell 61: 279-291.

Perkins, K.K., A. Admon, N. Patel, and R. Tjian. 1990. The Drosophila Fos-related AP-1 protein is a developmentally regulated transcription factor. Genes Dev. 4: 822-834.

Ptashne, M. 1988. How eukaryotic transcriptional activators work. Nature 335: 683-689.

Roux, E., M. Strubin, O. Hagenbüchle, and P.K. Wellauer. 1989. The cell-specific transcription factor PTF1 contains two different subunits that interact with DNA. Genes Dev. 3: $1613-1624$.

Rusconi, S., Y. Severne, O. Georgiev, I. Galli, and S. Wieland. 1990. A novel expression assay to study transcriptional acti- 
vators. Gene 89: 211-230.

Sambrook, J., E.F. Fritsch, and T. Maniatis. 1989. Molecular cloning: A laboratory manual. Cold Spring Harbor Laboratory Press, Cold Spring Harbor, New York.

Sanger, F., S. Nicklen, and A.R. Coulson. 1977. DNA sequencing with chain-terminating inhibitors. Proc. Natl. Acad. Sci. 74: 5463-5467.

Schaffner, W. 1989. How do transcription factors binding to the same DNA sequence sort out their jobs? Trends Genet. 5: $37-39$.

Schütte, J., J. Viallet, M. Nau, S. Segal, J. Fedorko, and J. Minna. 1989. jun- $B$ inhibits and $c$-fos stimulates the transforming and trans-activating activities of c-jun. Cell 59: 987-997.

Sierra, F., F. Tamone, C.R. Mueller, and U. Schibler. 1990. Differential in vitro transcription from the promoter of a rat alpha $2 \mu$ globulin gene in liver and spleen nuclear extracts. Mol. Biol. Med. 7: 131-146.

Singh, H., J.H. LeBowitz, A.S. Baldwin, and P.A. Sharp. 1988. Molecular cloning of an enhancer binding protein: Isolation by screening of an expression library with a recognition site DNA. Cell 52: 415-423.

Studier, F.W., A.H. Rosenberg, and J.J. Dunn. 1990. Use of T7 RNA polymerase to direct the expression of cloned genes. Methods Enzymol. (in press).

Tanaka, M. and W. Herr. 1990. Differential transcriptional activation by oct- 1 and oct-2: Interdependent activation domains induce oct-2 phosphorylation. Cell 60: 375-386.

Vinson, C.R., K.L. LaMarco, P.F. Johnson, W.H. Landschulz, and S.L. McKnight. 1988. In situ detection of sequence-specific DNA binding activity specified by a recombinant bacteriophage. Genes Dev. 2: 801-806.

Zerial, M.L., L. Toschi, R.P. Ryseck, M. Schuermann, and R. Müller. 1989. The product of a novel growth factor activated gene, fos $B$, interacts with JUN proteins enhancing their DNA binding activity. EMBO I. 8: 805-819. 


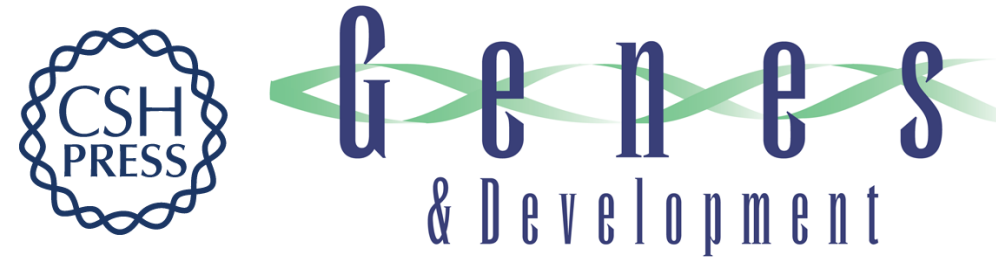

\section{LAP, a novel member of the C/EBP gene family, encodes a liver-enriched transcriptional activator protein.}

P Descombes, M Chojkier, S Lichtsteiner, et al.

Genes Dev. 1990, 4:

Access the most recent version at doi:10.1101/gad.4.9.1541

References This article cites 32 articles, 14 of which can be accessed free at: http://genesdev.cshlp.org/content/4/9/1541.full.html\#ref-list-1

License

Email Alerting

Service

Receive free email alerts when new articles cite this article - sign up in the box at the top right corner of the article or click here.

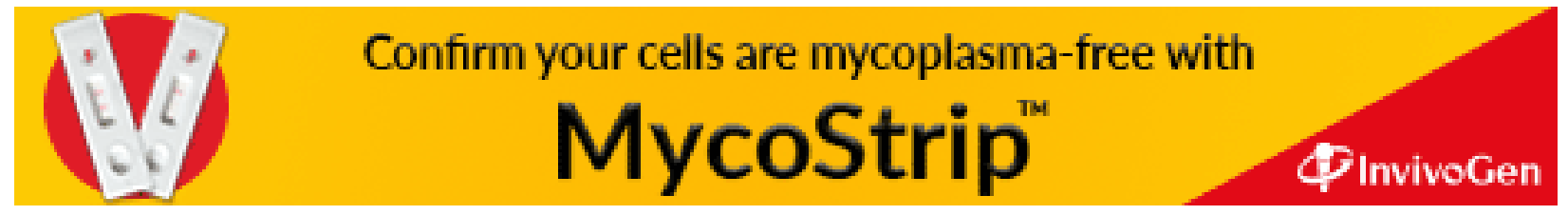

